\title{
Hospitalization Outcomes and Comorbidities of Bulimia Nervosa: A Nationwide Inpatient Study
}

Rikinkumar S. Patel ${ }^{1}$, Baris Olten ${ }^{2}$, Priya Patel ${ }^{3}$, Kaushal Shah ${ }^{4}$, Zeeshan Mansuri ${ }^{5}$

1. Department of Global Public Health, Arcadia University, Philadelphia, USA 2. Yale Child Research Center, Yale University School of Medicine 3. Department of Psychiatry, Windsor University School of Medicine, Philadelphia, USA 4. Psychiatry and Behavioral Health, Chicago Lakeshore Hospital 5. Psychiatry, Texas Tech University Health Sciences Center at Odessa/permian Basin

Corresponding author: Rikinkumar S. Patel, rpatel_09@arcadia.edu

\section{Abstract}

Objective

To evaluate inpatient outcomes and the prevalence of psychiatric and medical comorbidities in bulimia nervosa.

Methods

We used the Nationwide Inpatient Sample (NIS) from the Healthcare Cost and Utilization Project (HCUP). We identified bulimia nervosa as the primary diagnosis and medical and psychiatric comorbidities using ICD9$\mathrm{CM}$ codes. The differences in comorbidities were quantified using the Chi-square $(\chi 2)$ test, and a multinomial logistic regression model was used to quantify associations among comorbidities (odds ratio $(\mathrm{OR})$ ).

Results

The sample consisted of 3,319 inpatient admissions with bulimia nervosa between 2010-2014. Overall, 88\% patients were younger than 40 years of age $(p<0.001)$. Bulimia nervosa was seen in a higher proportion of females (92.5\%). The mean inpatient stay was 9.15 days and had a variable trend, whereas inpatient charges have been increasing $(\mathrm{p}<0.001)$, averaging $\$ 34,398$ (USD). The odds of having a longer hospitalization $>7$ days (median) was seen in patients with comorbid fluid/electrolyte disorders $(\mathrm{OR}=1.816 ; \mathrm{p}<0.001)$ and comorbid depression $(\mathrm{OR}=1.745 ; \mathrm{p}<0.001)$. The most prevalent psychiatric comorbidities were psychosis (52.4\%), followed by depression (23.5\%). Females had three times higher odds of comorbid diabetes (OR = 3.374; $\mathrm{p}<0.001$ ), hypertension ( $\mathrm{OR}=2.548$; $\mathrm{p}$-value $<0.001)$, comorbid depression $(\mathrm{OR}=1.670 ; \mathrm{p}=0.002)$, and drug abuse $(\mathrm{OR}=2.008 ; \mathrm{p}<0.001)$.

Received 04/26/2018

Review began 04/26/2018 Review ended 04/30/2018 Published 05/05/2018

\section{() Copyright 2018}

Patel et al. This is an open access article distributed under the terms of the Creative Commons Attribution License CC-BY 3.0., which permits unrestricted use, distribution, and reproduction in any medium, provided the original author and source are credited.
Conclusion

Our study established psycho-socio-demographic characteristics, hospitalization outcomes, and comorbidities of bulimia nervosa patients. We believe that medical and psychiatric comorbidities of bulimia nervosa should be carefully investigated by clinicians as they can further complicate the management of bulimia nervosa and result in adverse inpatient outcomes.

Categories: Internal Medicine, Psychiatry, Quality Improvement

Keywords: bulimia, eating disorders, comorbidities, national inpatient sample, inpatient psychiatry, demographics

\section{Introduction}

Bulimia nervosa is a potentially life-threatening eating disorder characterized by recurrent episodes of binge eating and inappropriate compensatory purging behaviors, such as self-induced vomiting, laxative abuse, enemas, and excessive exercise [1]. World Health Organization World Mental Health Surveys estimated that the lifetime prevalence of bulimia nervosa is $0.8 \%$ and the median age of onset is in the late teens to the early twenties [2]. In the United States, the lifetime prevalence of bulimia nervosa in men and women was estimated to be $0.5 \%$ and $1.5 \%$, respectively [3]. Bulimia nervosa has a significant impact on psychosocial functioning. In the United States, impaired psychosocial functioning was found among $78 \%$ of the patients; $44 \%$ reporting severe impairment.

Patients with bulimia nervosa usually have a lifetime history of many psychiatric disorders that were shown to be associated with a poorer prognosis [4]. The most common psychiatric co-morbidities of bulimia nervosa are unipolar major depression (50\%), specific phobia (50\%), post-traumatic stress disorder (PTSD) (45\%), attention-deficit/hyperactivity disorder (ADHD) (35\%) and alcohol use disorder (34\%) [3]. Identifying and 
addressing the psychiatric co-morbidities of bulimia nervosa are crucial steps in the management of this disease as these co-morbidities can further decrease the psychosocial functioning. Suicide attempts are common with bulimia nervosa and were estimated to occur in $17 \%$ of the patients [5]. Successful treatment of bulimia nervosa can often resolve anxiety and depressive disorders [4]. Likewise, identifying and managing comorbid psychiatric disorders improve the treatment adherence and the outcome of treatment of bulimia nervosa.

Medical complications in patients with bulimia nervosa are common and can affect many organ systems, such as gastrointestinal, renal, cardiac, and endocrine [6]. Medical complications can contribute to a reduced quality of life and increased rates of mortality associated with bulimia nervosa. Compared to the general population, all-cause mortality is two to eight times greater in patients with bulimia nervosa [7]. Inpatient treatment of bulimia nervosa can be indicated in patients that have severe symptoms, psychiatric co-morbidities, or patients who develop serious medical complications [8].

In this inpatient sample-based retrospective study, we demonstrated the estimation of inpatient admission outcomes, as well as the rates of comorbid psychiatric and medical disorders in admitted bulimia nervosa patients. To the best of our knowledge, this is the first cohort retrospective study that investigates the inpatient psycho-socio-demographic characteristics and admission outcomes of bulimia nervosa patients.

\section{Materials And Methods \\ Data source}

In this study, a retrospective analysis was performed using the Healthcare Cost and Utilization Project's (HCUP) Nationwide Inpatient Sample (NIS) data [9]. The Agency for Healthcare Research and Quality (AHRQ) sponsors the HCUP databases that categorize trends in hospital utilization and cost across the United States. The HCUP-NIS database is the largest inpatient database as it includes 4,411 non-federal community hospitals from 45 states in the United States. We weighted the estimated samples to reduce the margin of error and to reproduce the results equivalent to all 50 states in the United States. In the NIS data, the privacy of individual patients, physicians, and hospitals, the state, and hospital identifiers are protected and de-identified. Sample non-clinical-related information is patient's demographic data that includes age, gender, race and primary payer status, hospital characteristics, and total charges. Sample clinical-related information includes principal and other diagnosis, comorbidities, disposition status and the length of inpatient stay [9]. As the NIS database does not contain patient identification, this study did not require Institution Review Board permission.

\section{Variables of interest}

The participants in this study were identified by the International Classification of Disease, Ninth Revision Clinical Modification (ICD-9-CM) codes with a principal diagnosis of bulimia nervosa at the time of admission. Bulimia nervosa was identified using the ICD-9-CM diagnosis code 307.51. To measure the differences in hospitalization outcomes in bulimia nervosa patients, the outcome variables of interest included the severity of morbidity that measures the loss of body functions, the risk of mortality that measures the likelihood of dying, and discharge status of the patient. The $3 \mathrm{M}^{\mathrm{TM}}$ All Patient Refined Diagnosis-related Groups (APR-DRGs) were assigned using software developed by the 3M Health Information Systems (3M Health Information Systems, Murray, UT). This severity measure includes the APR-DRG, the severity of morbidity, and the risk of mortality within each base APR-DRG [9]. We calculated the length of the inpatient stay as the number of nights the patient remained in the hospital for a particular discharge. The length of inpatient stay in this analysis was all-cause. The total inpatient charges did not include professional fees and non-covered charges. The principal diagnosis had been identified at the time of admission, whereas we recorded comorbidities throughout the entire inpatient stay. By a common definition, comorbidities were considered coexisting conditions with bulimia nervosa, the principal disorder under this study. The Agency for Healthcare Research and Quality (AHRQ) comorbidity software was used to generate binary variables that identified various psychiatric and medical comorbidities in the discharge records using ICD-9-CM codes [10]. The ICD-9-CM codes used to identify comorbidities associated with bulimia nervosa are mentioned in Table 1 . 


\section{Cureus}

\begin{tabular}{|l|l}
\hline Comorbidity & ICD-9-CM Diagnosis Code \\
\hline Congestive heart failure & $398.91,402.01,402.11,402.91,404.01,404.03,404.11,404.13,404.91,404.93,428.0-428.9$ \\
\hline $\begin{array}{l}\text { Hypertension } \\
\text { Diabetes without chronic }\end{array}$ & $401.1,401.9,642.00-642.04,401.0,402.00-405.99,437.2,642.10-642.24,642.70-642.94$ \\
complications & \\
Diabetes with chronic & $249.40-249.91,250.40-250.93,775.1$ \\
complications & $243-244.2,244.8,244.9$ \\
\hline Hypothyroidism & $403.01,403.11,403.91,404.02,404.03,404.12,404.13,404.92,404.93,585.3,585.4,585.5,585.6,585.9$, \\
\hline Renal failure & $586, \mathrm{~V} 42.0, \mathrm{~V} 45.1, \mathrm{~V} 45.11, \mathrm{~V} 45.12, \mathrm{~V} 56.0-\mathrm{V} 56.32, \mathrm{~V} 56.8$ \\
\hline Obesity & $278.0,278.00,278.01,278.03,649.10-649.14,793.91, \mathrm{~V} 85.30-\mathrm{V} 85.39, \mathrm{~V} 85.41-\mathrm{V} 85.45, \mathrm{~V} 85.54$ \\
\hline Weight Loss & $260-263.9,783.21,783.22$ \\
\hline $\begin{array}{l}\text { Fluid and electrolyte } \\
\text { disorders }\end{array}$ & $276.0-276.9$ \\
\hline Deficiency anemias & $280.1-281.9,285.21-285.29,285.9$ \\
\hline Alcohol abuse & $291.0-291.3,291.5,291.8,291.81,291.82,291.89,291.9,303.00-303.93,305.00-305.03$ \\
\hline Drug abuse & $292.0,292.82-292.89,292.9,304.00-304.93,305.20-305.93,648.30-648.34$ \\
\hline $\begin{array}{l}\text { Psychosis } \\
\text { Depression }\end{array}$ & $295.00-298.9,299.10,299.11$ \\
\hline
\end{tabular}

TABLE 1: International Classification of Disease, Ninth Clinical Modification (ICD-9-CM) Codes Used to Identify Comorbidities of Bulimia Nervosa

\section{Approaches}

We used the Statistical Package for the Social Science (SPSS), version 23.0 (IBM SPSS Statistics, Armonk, NY) to conduct a retrospective analysis over the HCUP-NIS database from 2010-2014 [11]. Descriptive statistics were used to summarize the results. The mean and standard deviations were used to explain the continuous variables. Pearson's chi-square test and independent sample T-test were used for categorical data and continuous data, respectively. On the other hand, the categorical variables were presented in percentage (\%) values. We used a multinomial logistic regression model to quantify associations among comorbidities and gender (odds ratio (OR); 95\% confidence interval (CI)), and the inpatient length of stay (OR; 95\% CI). We applied the discharge weight, which was given in the NIS database to attain national representation of the inpatient data. A p-value $<0.05$ was used as a reference to determine the statistical significance test result.

\section{Results}

\section{Demographic characteristics of bulimia nervosa}

The sample consisted of 3,319 inpatient admissions with bulimia nervosa listed as the primary diagnosis between $2010-2014$. Overall, $46.5 \%$ patients were aged 21 - 40 years and $41.5 \%$ were under 20 years; thus, approximately $88 \%$ of bulimia nervosa patients were younger than 40 years of age (p-value $<0.001$ ). On the contrary, bulimia nervosa was seen in $11.9 \%$ patients above 40 years' age. Bulimia nervosa was seen in a higher proportion of females compared to the males $(92.5 \%$ vs. $7.5 \%$, respectively; $p$-value $<0.001)$. The trend of bulimia nervosa from 2010-2014 was distributed according to patient's age group, as shown in Figure 1 with gender as shown in Figure 2. 


\section{Cureus}

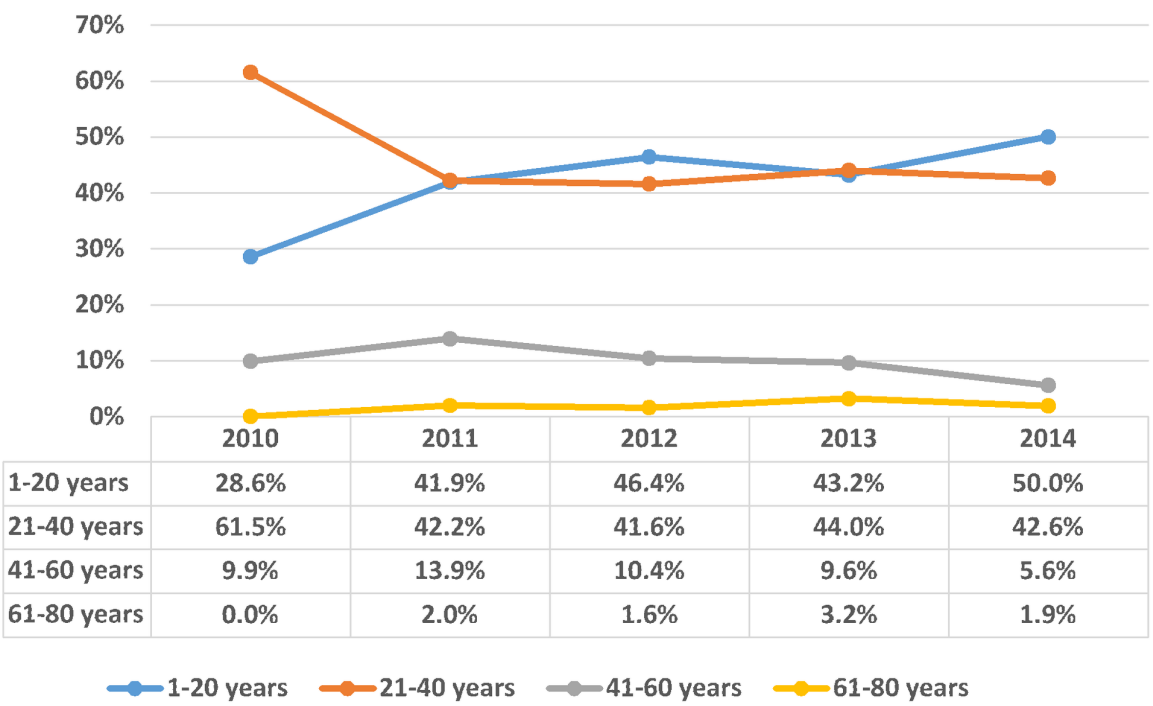

FIGURE 1: Trend of bulimia nervosa distributed according to age group

$\mathrm{X}$-axis: years; $\mathrm{Y}$-axis: proportion in percentage (\%). Significant $\mathrm{p}$-values $\leq 0.05$ at $95 \%$ confidence interval

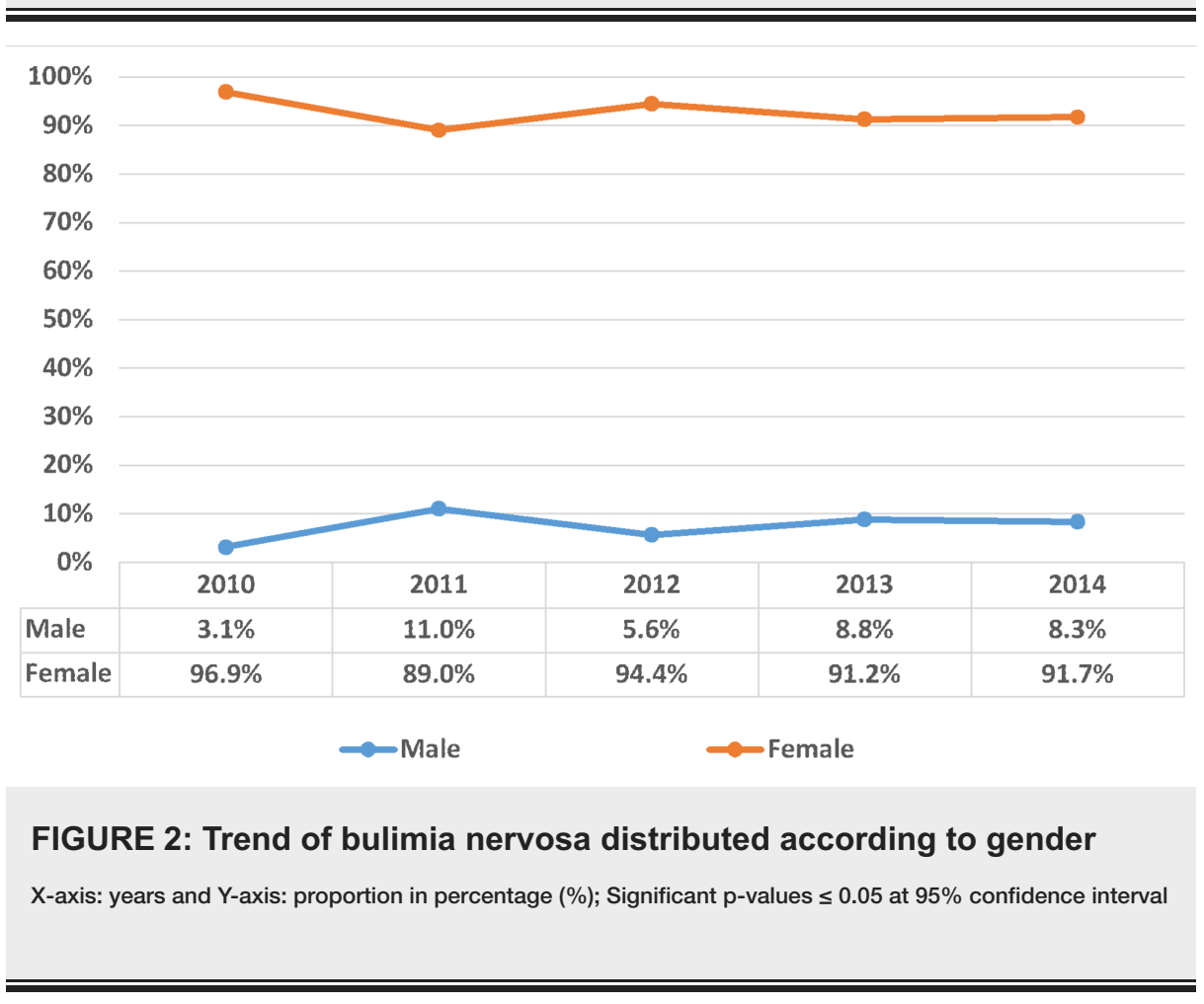

A total of 2,457 bulimia nervosa patients were White, which comprises $81.4 \%$ of the total, followed by Hispanic (6.7\%), Black (5\%), Asian (2.3\%), Native American (1.2\%), and others (3.5\%). The majority of the inpatient admissions for bulimia nervosa were covered by private insurance (55.7\%) and Medicaid (23.8\%).

The trend of bulimia nervosa from 2010-2014 was distributed according to the patients' race as shown in Figure 3. 


\section{Cureus}

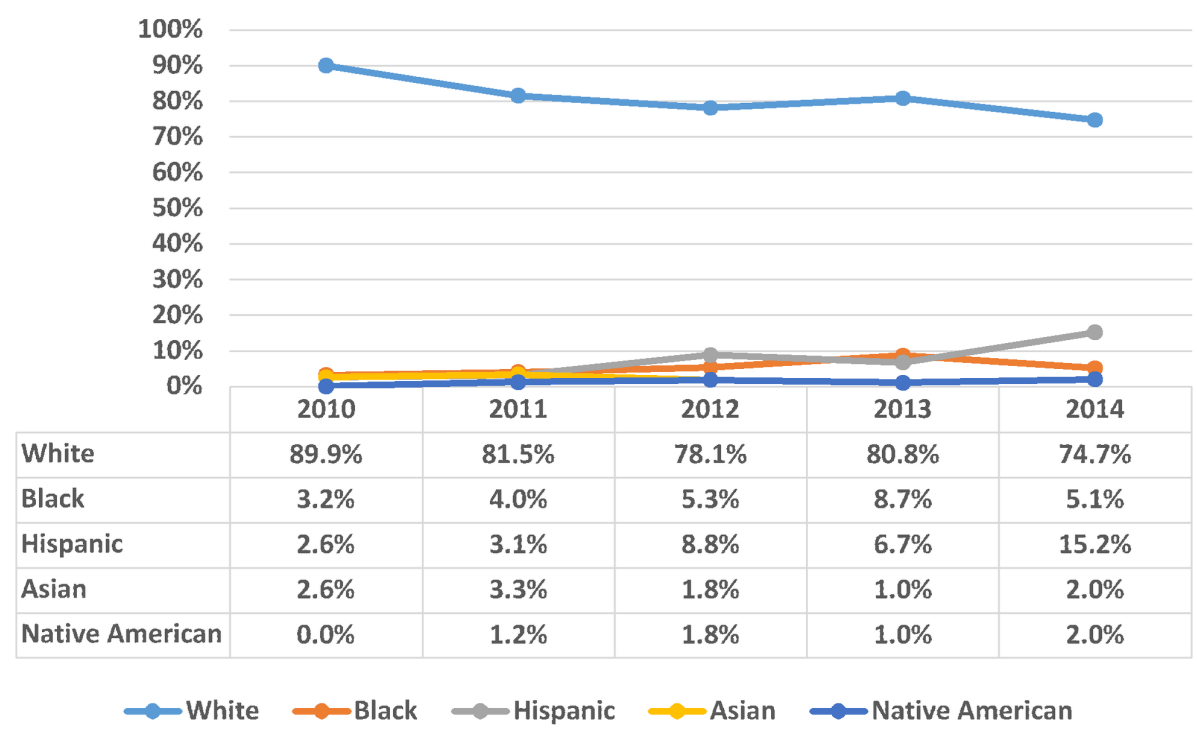

FIGURE 3: Trend of bulimia nervosa distributed according to race within bulimia nervosa patients

$\mathrm{X}$-axis: years; $\mathrm{Y}$-axis: proportion in percentage (\%). Significant $\mathrm{p}$-values $\leq 0.05$ at $95 \%$ confidence interval

\section{Inpatient outcomes in bulimia nervosa}

A total of 2,428 patients (73.3\%) were hospitalized based on non-elective admission and 253 patients (7.7\%) with a principal diagnosis of bulimia nervosa were transferred in from an acute care facility. The mean length of stay in the hospital was 9.15 days (standard deviation $(\mathrm{SD})=7.80$; $\mathrm{p}$-value $<0.001$ ), and the mean inpatient total charge was $\$ 34,398$ USD ( $S D=37692.19$; $p$-value $<0.001$ ). The trend of bulimia nervosa was distributed according to the mean inpatient stay and mean inpatient charges, as shown in Figure 4. During the period 2010-2014, there were a total of 30,367 inpatient days and \$113 million USD inpatient costs due to bulimia nervosa in the United States.

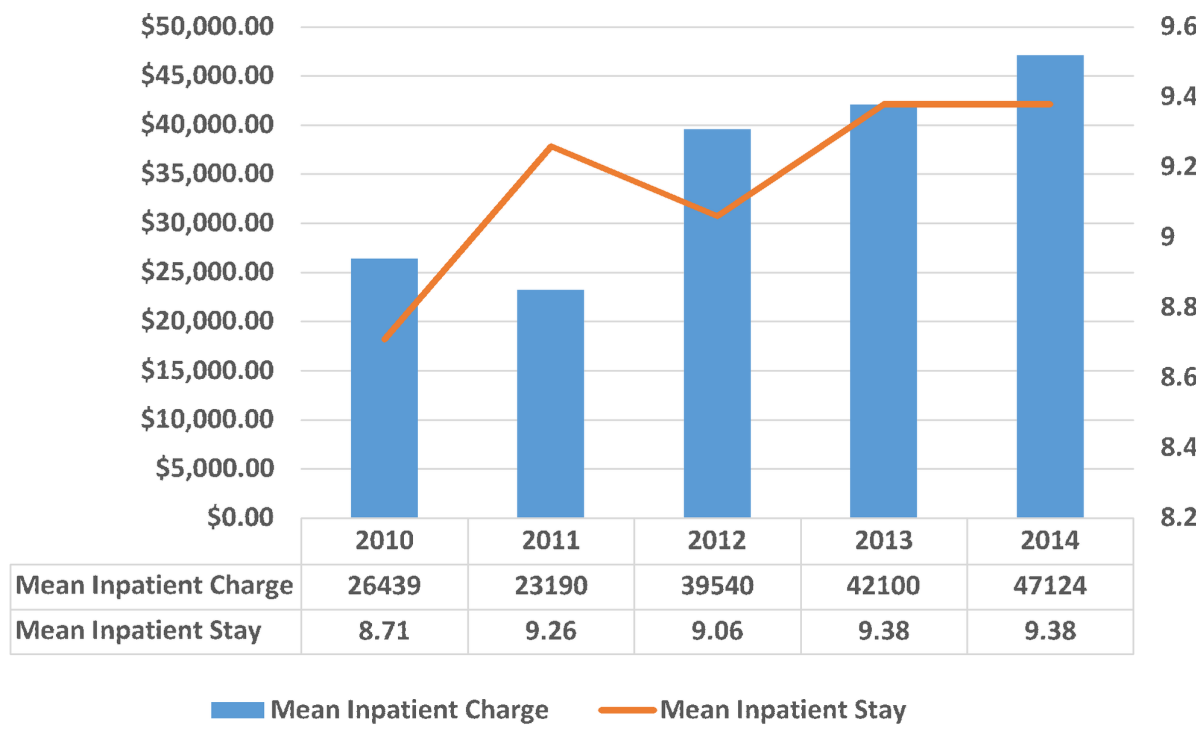

FIGURE 4: Trend of bulimia nervosa distributed according to the mean inpatient stay and mean inpatient charges

X-axis: years; Left Y-axis: mean inpatient charge (in USD); Right Y-axis: mean inpatient stay (in days). Significant $p$-values $\leq 0.05$ at $95 \%$ confidence interval.

USD: United States dollars 


\section{Cureus}

About, $71.5 \%$ (number $(\mathrm{N})=2,375$ ) of the bulimia nervosa patients had minor to moderate severity of morbidity and $98 \%(\mathrm{~N}=3,254)$ patients were sub-classified as minor to moderate risk of mortality. There was no bulimia nervosa-related inpatient deaths between 2010-2014 in the United States hospitals. Figure 5 shows the severity of morbidity and risk of mortality in the bulimia nervosa patients.
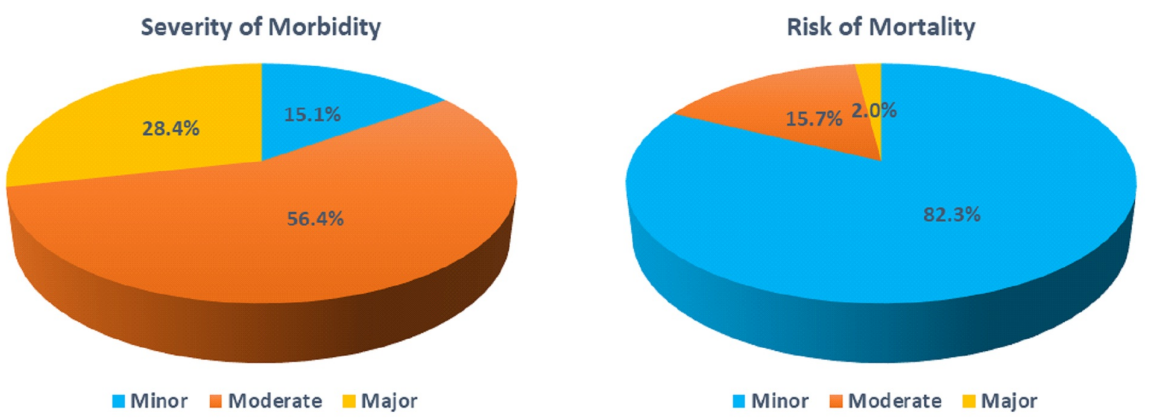

FIGURE 5: Distribution of bulimia nervosa according to the severity of morbidity and risk of mortality

Significant $p$-values $\leq 0.05$ at $95 \%$ confidence interval

Among adverse discharge outcomes of bulimia nervosa patients, there was a variable trend in the disposition to a short-term hospital $(2.4 \% ; \mathrm{N}=80)$ and a skilled nursing facility (SNF)/intermediate care facility (ICF) $(8.6 \% ; \mathrm{N}=286)(\mathrm{p}<0.001)$. The trend of bulimia nervosa from $2010-2014$ was distributed according to discharge, as shown in Figure 6.

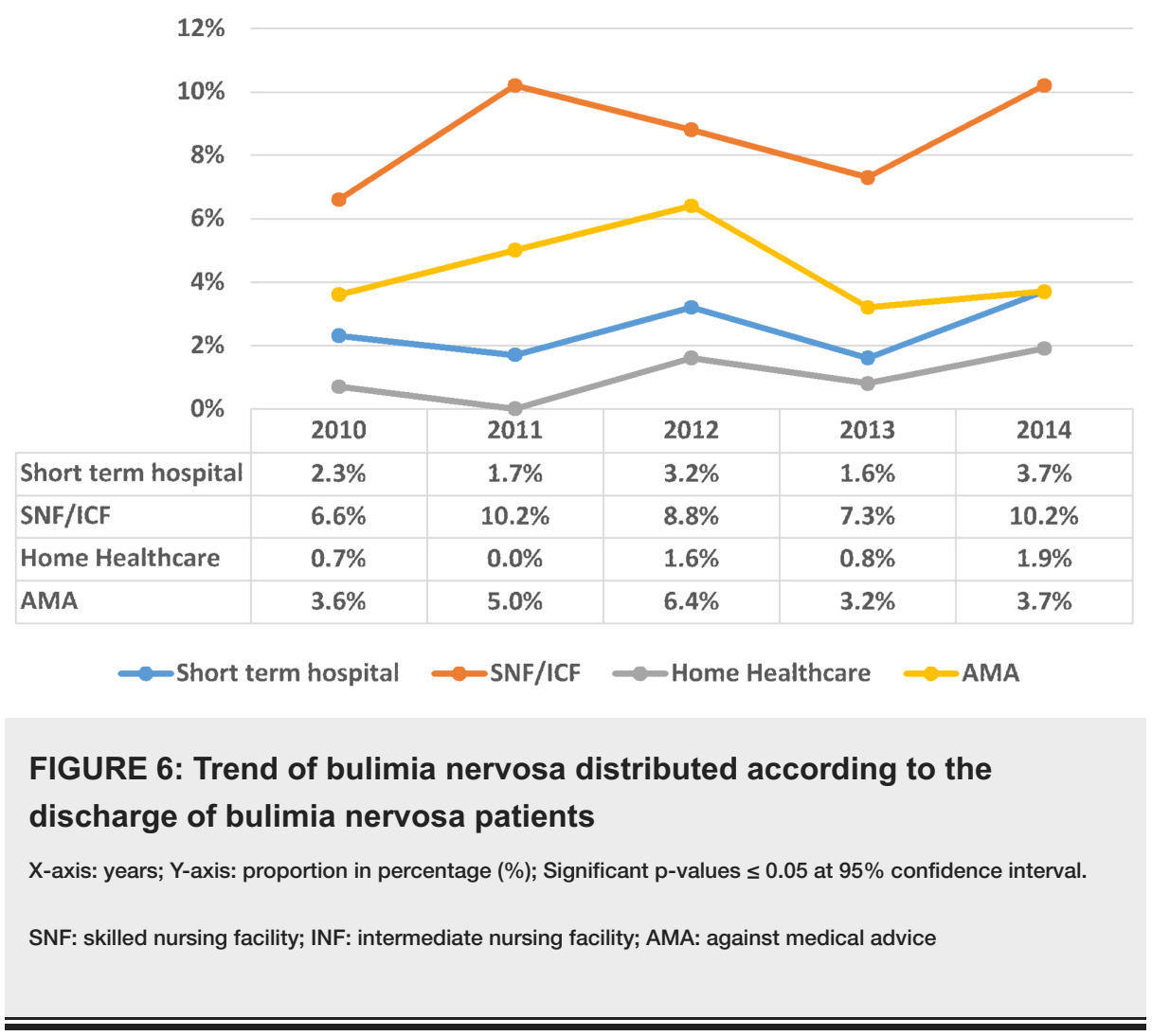

\section{Comorbidities in bulimia nervosa}

The most prevalent psychiatric comorbidities present in bulimia nervosa were psychosis ( $N=1740 ; 52.4 \%)$, followed by depression $(\mathrm{N}=779 ; 23.5 \%)$. Drug abuse $(\mathrm{N}=715 ; 21.5 \%)$ and alcohol abuse $(\mathrm{N}=566 ; 17.1 \%)$ were less prevalent psychiatric comorbidities in the admitted bulimia nervosa patients. 
The most prevalent medical comorbidity present in bulimia nervosa was fluid and electrolyte disorders $(\mathrm{N}=$ $1,214 ; 36.6 \%)$. The other medical co-morbidities reported among admitted patients were weight loss $(\mathrm{N}=$ $740 ; 22.3 \%)$, deficiency anemias $(\mathrm{N}=354 ; 10.4 \%)$, hypothyroidism $(\mathrm{N}=248 ; 7.5 \%)$, obesity $(\mathrm{N}=177 ; 5.3 \%)$, hypertension $(\mathrm{N}=190 ; 5.7 \%)$, uncomplicated diabetes $(\mathrm{N}=99 ; 3 \%)$, diabetes with chronic complications $(\mathrm{N}=$ $48 ; 1.4 \%)$, renal failure ( $\mathrm{N}=34 ; 1 \%)$, and congestive heart failure $(\mathrm{N}=10 ; 0.3 \%)$. The prevalence of psychiatric and medical comorbidities in bulimia nervosa patients is shown in Figure 7.

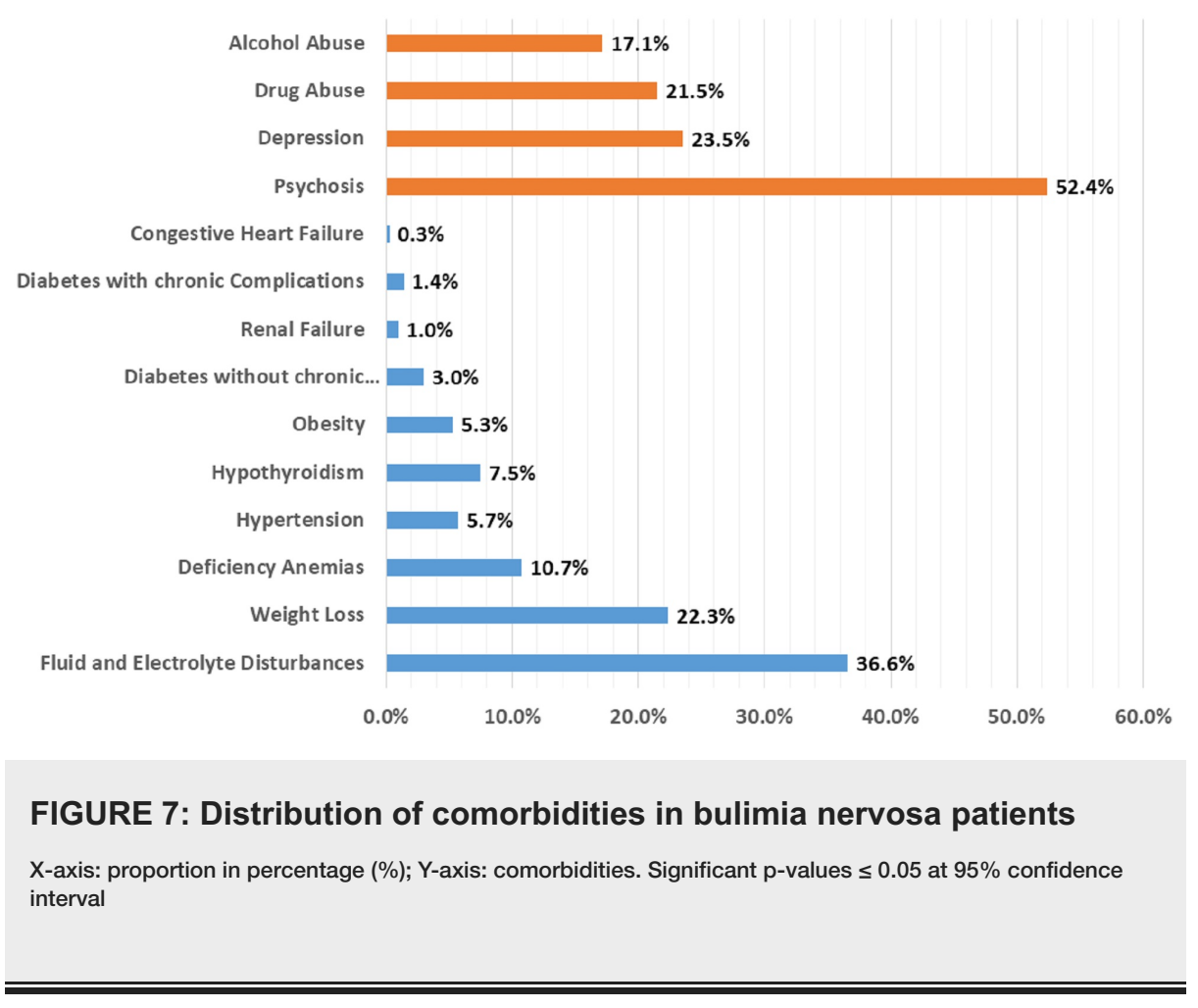

Among chronic condition comorbidities, female patients had three times higher odds of comorbid uncomplicated diabetes $(\mathrm{OR}=3.374 ; 95 \%$ CI 1.899 - 5.994; $\mathrm{p}$-value < 0.001), followed by hypertension $(\mathrm{OR}=$ 2.548; 95\% CI 1.582 - 4.103; p-value < 0.001). Also, females had higher odds of psychiatric conditions, like comorbid depression $(\mathrm{OR}=1.670 ; 95 \% \mathrm{CI} 1.199-2.326$; $\mathrm{p}$-value $=0.002)$ and drug abuse $(\mathrm{OR}=2.008$; $95 \% \mathrm{CI}$ 1.477 - 2.731; $\mathrm{p}$-value $<0.001)$. Bulimia nervosa females also had a higher risk of fluid and electrolyte disorders during hospitalization $(\mathrm{OR}=1.437 ; 95 \%$ CI $1.089-1.895 ; \mathrm{p}$-value $=0.010)$.

The median length of inpatient stay of bulimia nervosa patients was seven days. The odds of having a longer hospitalization with a stay of more than seven days was seen in patients with comorbid fluid and electrolyte disorders $(\mathrm{OR}=1.816 ; 95 \% \mathrm{CI} 1.557-2.118 ; \mathrm{p}$-value $<0.001)$, comorbid depression $(\mathrm{OR}=1.745 ; 95 \% \mathrm{CI}$ 1.436 - 2.120; $p$-value < 0.001), and comorbid drug abuse $(\mathrm{OR}=1.192 ; 95 \%$ CI $0.996-1.426$; $\mathrm{p}$-value $=0.044)$. The odds of a longer hospitalization stay were also seen in patients with comorbid congestive heart failure but the results were not statistically significant $(\mathrm{OR}=2.079 ; 95 \%$ CI $0.564-7.658$; $\mathrm{p}$-value $=0.272)$.

\section{Discussion}

In our retrospective inpatient sample cohort study, we demonstrated that most of the admitted bulimia nervosa patients were White women who were under 40 years of age. The mean length of hospital stay was nine days, which is lower than the reported average inpatient stay in affective and psychotic disorders [1214]. More than $70 \%$ of the admitted patients had a moderate morbidity. The odds of having a longer hospitalization stay was seen in bulimia nervosa patients with comorbid fluid and electrolyte disorders, comorbid depression, and comorbid drug abuse. There were no bulimia nervosa-related inpatient deaths between 2010-2014, which is not surprising given that the likelihood of dying was reported to be minor among $82.3 \%$ of the admitted patients. The present study demonstrated the pervasiveness of psychiatric and medical comorbidities in patients admitted with a diagnosis of bulimia nervosa. The most prevalent psychiatric and medical comorbidities were psychosis and fluid and electrolyte disturbances, respectively.

A nationally representative survey conducted in the United States reported that $95 \%$ of the patients with bulimia nervosa had at least one comorbid disorder and $64 \%$ had three or more comorbid disorders [3]. In our study, psychosis, depression, and drug abuse emerged as the most common psychiatric comorbidities of bulimia nervosa in admitted patients. Unipolar major depression is commonly observed in patients with bulimia nervosa and usually begins after the onset of bulimia nervosa [3, 15]. The lifetime rate for unipolar depression in patients with bulimia nervosa (79\%) exceeds the rate in the general population (23\%) [15-16]. 
Previous research has shown that emotional dysregulation often occurs in bulimia nervosa, which might contribute to the bulimia nervosa patient's propensity for depression [17]. Patients with bulimia nervosa are prone to impulsive or compulsive injury (skin cutting or burning with a lit cigarette) [18]. A study found that all-cause mortality in bulimia nervosa was roughly three times greater than all-cause mortality for bipolar disorder and depression. However, as per our study, there was no reported inpatient death among patients with bulimia nervosa between 2010-2014. The comorbidity of bulimia nervosa with psychosis is less investigated than their comorbidity with mood and anxiety disorders. A previous study that examined psychosis in patients with bulimia nervosa did not report any case of comorbid schizophrenia, although symptoms of psychosis were reported [19]. Major depression is the most common comorbidity, followed by anxiety disorders, including generalized anxiety disorder, panic disorder, obsessive-compulsive disorder, social phobia, and posttraumatic stress disorder in nearly $60 \%$ of bulimia nervosa patients. The onset of bulimia classically occurs in adolescence or young adulthood [20]. Comorbid psychosis in young adults and adolescents related to such anxiety disorders is common, so it is very rare to have psychosis because of schizophrenia that was observed with bulimia nervosa patients in our study. The lifetime prevalence of an alcohol use disorder in patients with bulimia nervosa was estimated to be $46 \%$ [21]. A previous study that investigated the neurobiological and clinical variables associated with alcohol abuse in bulimia nervosa suggested that hypercortisolism and depressive symptoms seen in patients with bulimia nervosa could explain the higher occurrence of alcohol use disorder in this patient population [22]. Illicit drug use in patients with bulimia nervosa was reported to be $26 \%$ in a national comorbidity survey [3]. A previous study that examined the rates of substance abuse in bulimia nervosa reported that the rate of substance use was elevated among women with a history of sexual or physical abuse as compared to women without such a history. The authors suggested that the higher occurrence of substance use in bulimia nervosa may not be related uniquely to the bulimia nervosa diagnostic status but may be related to characteristics shared by women with bulimia nervosa, such as a history of sexual or physical abuse [23]. In our study, we reported females with bulimia nervosa had higher odds of psychiatric conditions, like comorbid depression and drug abuse.

Medical complications of bulimia nervosa depend upon the method and frequency of purging [24]. Selfinduced vomiting was reported to cause most medical complications. As per our study, the most commonly reported medical comorbidities were electrolyte disturbances, weight loss, and deficiency anemias. The most common medical complications of bulimia nervosa related to electrolyte disturbances include dehydration, hypokalemia, hyponatremia, and metabolic alkalosis [25]. Hypokalemia in otherwise healthy young adults is highly specific for bulimia nervosa and hypokalemia-related cardiac arrhythmias can explain the increased mortality rate observed in patients with bulimia nervosa [26]. Observed weight loss in patients with bulimia nervosa might result from the high frequency of vomiting seen in these patients [27]. In our study, among medical comorbidities, female patients had higher odds of comorbid uncomplicated diabetes, hypertension, and electrolyte disturbances.

This dataset is subject to minimal reporting bias, and all information is coded independently of the individual practitioner, making it a potentially more reliable source. The limitation for using hospitalization (and not the patient) as the unit of analysis is that it does not translate to generalizability for all patients with bulimia nervosa. There may have been underreporting of chronic comorbidities in the NIS data because the administrative database was used. Hence, clinical data were not incorporated in the data source. We recommend that future research examine the influence of psychiatric comorbidities with clinical data. In addition to that, we recommend that future research should investigate the impact of different medical and psychiatric comorbidities of bulimia nervosa on admission outcomes.

\section{Conclusions}

Our study established the psycho-socio-demographic characteristics and hospitalization outcomes of bulimia nervosa patients, including disease severity, mortality, length of inpatient stay, and total inpatient charges. In addition to that, we demonstrated the rates of the most common medical and psychiatric comorbidities of bulimia nervosa. Patients with bulimia nervosa can present with medical complications and attempt to hide their eating disorder from the clinicians. We believe that medical and psychiatric comorbidities of bulimia nervosa should be carefully investigated by clinicians as they can further complicate the management of bulimia nervosa and result in adverse inpatient outcomes.

\section{Additional Information \\ Disclosures}

Human subjects: Consent was obtained by all participants in this study. N/A issued approval N/A. A retrospective analysis was performed using the Healthcare Cost and Utilization Project's (HCUP) Nationwide Inpatient Sample (NIS) data. As the NIS database does not contain patient identification, this study did not require Institution Review Board permission. Animal subjects: All authors have confirmed that this study did not involve animal subjects or tissue. Conflicts of interest: In compliance with the ICMJE uniform disclosure form, all authors declare the following: Payment/services info: All authors have declared that no financial support was received from any organization for the submitted work. Financial relationships: All authors have declared that they have no financial relationships at present or within the previous three years with any organizations that might have an interest in the submitted work. Other relationships: All authors 
have declared that there are no other relationships or activities that could appear to have influenced the submitted work.

\section{References}

1. American Psychiatric Association: Diagnostic and Statistical Manual of Mental Disorders (5th ed.) . American Psychiatric Association (ed): American Psychiatric Publishing, Washington, DC; 2013. 10.1176/appi.books.9780890425596

2. Kessler RC, Berglund PA, Chiu WT, et al.: The prevalence and correlates of binge eating disorder in the World Health Organization World Mental Health Surveys. Biol Psychiatry. 2013, 73:904-14. 10.1016/j.biopsych.2012.11.020

3. Hudson JI, Hiripi E, Pope HG Jr, Kessler RC: The prevalence and correlates of eating disorders in the National Comorbidity Survey Replication. Biol Psychiatry. 2007, 61:348-58. 10.1016/j.biopsych.2006.03.040

4. Milos GF, Spindler AM, Buddeberg C, Crameri A: Axes I and II comorbidity and treatment experiences in eating disorder subjects. Psychother Psychosom. 2003, 72:276-85. 10.1159/000071899

5. Yao S, Kuja-Halkola R, Thornton LM, et al.: Familial liability for eating disorders and suicide attempts: evidence from a population registry in Sweden. JAMA Psychiatry. 2016, 73:284-91. 10.1001/jamapsychiatry.2015.2737

6. Mitchell JE, Crow S: Medical complications of anorexia nervosa and bulimia nervosa. Curr Opin Psychiatry. 2006, 19:438-43. 10.1097/01.yco.0000228768.79097.3e

7. Arcelus J, Mitchell AJ, Wales J, Nielsen S.: Mortality rates in patients with anorexia nervosa and other eating disorders. A meta-analysis of 36 studies. Arch Gen Psychiatry. 2011, 68:724-31. 10.1001/archgenpsychiatry.2011.74

8. Rø Ø, Martinsen EW, Hoffart A, Rosenvinge JH: Short-term follow-up of severe bulimia nervosa after inpatient treatment. Eur Eat Disorders Rev. 2003, 11:405-17. 10.1002/erv.533

9. NIS Database Documentation. (2018). Accessed: April 15, 2018: http://www.hcupus.ahrq.gov/db/nation/nis/nisdbdocumentation.jsp.

10. HCUP Tools and Software. (2018). Accessed: April 15, 2018: http://www.hcupus.ahrq.gov/tools_software.jsp.

11. Acosta MC, Possemato K, Maisto SA, et al.: Web-delivered CBT reduces heavy drinking in OEF-OIF veterans in primary care with symptomatic substance use and PTSD. Behav Ther. 2017, 48:262-76. 10.1016/j.beth.2016.09.001

12. Sitta P, Brand S, Schneider F, et al.: Duration of inpatient depression treatment--fair benchmarking between hospitals (Article in German). Psychother Psychosom Med Psychol. 2006, 56:128-37. 10.1055/s2005-915331

13. Gonzalez-Pinto AM, Dardennes R, Zélicourt M, et al.: In-patient care costs of patients with bipolar I disorder: a comparison between two European centers. J Affect Disord. 2010, 121:152-55. 10.1016/j.jad.2009.05.010

14. van Rensburg AB, Olorunju S: Diagnosis and treatment of schizophrenia in a general hospital based acute psychiatric ward. Afr J Psychiatry (Johannesbg). 2010, 13:204-10.

15. Fernandez-Aranda F, Pinheiro AP, Tozzi F, et al.: Symptom profile of major depressive disorder in women with eating disorders. Aust N Z J Psychiatry. 2007, 41:24-31. 10.1080/00048670601057718

16. Kessler RC, Berglund P, Demler O, et al.: Lifetime prevalence and age-of-onset distributions of DSM-IV disorders in the National Comorbidity Survey Replication. Arch Gen Psychiatry. 2005, 62:593-602. 10.1001/archpsyc.62.6.593

17. Lavender JM, Wonderlich SA, Engel SG, et al.: Dimensions of emotion dysregulation in anorexia nervosa and bulimia nervosa: a conceptual review of the empirical literature. Clin Psychol Rev. 2015, 40:111-22. 10.1016/j.cpr.2015.05.010

18. Cucchi A, Ryan D, Konstantakopoulos G, et al.: Lifetime prevalence of non-suicidal self-injury in patients with eating disorders: a systematic review and meta-analysis. Psychol Med. 2016, 46:1345-58. 10.1017/S0033291716000027

19. Miotto P, Pollini B, Restaneo A, et al.: Symptoms of psychosis in anorexia and bulimia nervosa . Psychiatry Res. 2010, 175:237-43. 10.1016/j.psychres.2009.03.011

20. Bulimia Nervosa Psychiatric Morbidity. (2006). Accessed: April 15, 2018: http://www.health.am/psy/more/bulimia_nervosa_int2/.

21. Bulik CM, Klump KL, Thornton L, et al.: Alcohol use disorder comorbidity in eating disorders: a multicenter study. J Clin Psychiatry. 2004, 65:1000-6. 10.4088/JCP.v65n0718

22. Vaz-Leal FJ, Ramos-Fuentes MI, Rodríguez-Santos L, et al.: Neurobiological and clinical variables associated with alcohol abuse in bulimia nervosa. Eur Eat Disord Rev. 2015, 23:185-92. 10.1002/erv.2352

23. Dohm FA, Striegel-Moore RH, Wilfley DE, et al.: Self-harm and substance use in a community sample of Black and White women with binge eating disorder or bulimia nervosa. Int J Eat Disord. 2002, 32:389-400. 10.1002/eat.10104

24. Forney KJ, Buchman-Schmitt JM, Keel PK, Frank GK: The medical complications associated with purging. Int J Eat Disord. 2016, 49:249-59. 10.1002/eat.22504

25. Mehler PS, Walsh K: Electrolyte and acid-base abnormalities associated with purging behaviors . Int J Eat Disord. 2016, 49:311-8. 10.1002/eat.22503

26. Westmoreland P, Krantz MJ, Mehler PS: Medical complications of anorexia nervosa and bulimia. Am J Med. 2016, 129:30-37. 10.1016/j.amjmed.2015.06.031

27. Gendall KA, Bulik CM, Sullivan PF, et al.: Body weight in bulimia nervosa. Eat Weight Disord. 1999, 4:15764. 10.1007/BF03339730 